\title{
Granulocyte-macrophage stimulating factor (GM-CSF) increases circulating dendritic cells but does not abrogate suppression of adaptive cellular immunity in patients with metastatic colorectal cancer receiving chemotherapy
}

\author{
Micaela Martinez ${ }^{1}$, Nadia Ono ${ }^{1}$, Marina Planutiene ${ }^{2}$, Kestutis Planutis², Edward L Nelson ${ }^{1}$ and
} Randall F Holcombe ${ }^{2^{*}}$

\begin{abstract}
Background: Advanced cancer and chemotherapy are both associated with immune system suppression. We initiated a clinical trial in patients receiving chemotherapy for metastatic colorectal cancer to determine if administration of GM-CSF in this setting was immunostimulatory.
\end{abstract}

Methods: Between June, 2003 and January, 2007, 20 patients were enrolled in a clinical trial (NCT00257322) in which they received $500 \mathrm{ug}$ GM-CSF daily for 4 days starting 24 hours after each chemotherapy cycle. There were no toxicities or adverse events reported. Blood was obtained before chemotherapy/GM-CSF administration and 24 hours following the final dose of GM-CSF and evaluated for circulating dendritic cells and adaptive immune cellular subsets by flow cytometry. Peripheral blood mononuclear cell (PBMC) expression of $\gamma$-interferon and T-bet transcription factor (Tbx21) by quantitative real-time PCR was performed as a measure of Th1 adaptive cellular immunity. Pre- and post-treatment (i.e., chemotherapy and GM-CSF) samples were evaluable for 16 patients, ranging from 1 to 5 cycles (median 3 cycles, 6 biologic sample time points). Dendritic cells were defined as lineage $(-)$ and MHC class II high (+).

Results: $73 \%$ of patients had significant increases in circulating dendritic cells of $\sim 3 x$ for the overall group (5.8\% to $13.6 \%, p=0.02)$ and $\sim 5 x$ excluding non-responders ( $3.2 \%$ to $14.5 \%, p<0.001)$. This effect was sustained over multiple cycles for approximately half of the responders, but tachyphylaxis over subsequent chemotherapy cycles was noted for the remainder. Treatment also led to a significant reduction in the proportion of circulating regulatory T-cells (Treg; $\mathrm{p}=0.0042$ ). PBMC Tbx21 levels declined by $75 \%$ following each chemotherapy cycle despite administration of GM-CSF ( $p=0.02$ ). PBMC $\gamma$-interferon expression, however was unchanged.

Conclusions: This clinical trial confirms the suppressive effects of chemotherapy on Th1 cellular immunity in patients with metastatic colorectal cancer but demonstrates that mid-cycle administration of GM-CSF can significantly increase the proportion of circulating dendritic cells. As the role of dendritic cells in anti-tumor immunity becomes better defined, GM-CSF administration may provide a non-toxic intervention to augment this arm of the immune system for cancer patients receiving cytotoxic therapy.

Trial Registration: ClinicalTrials.gov: NCT00257322

\footnotetext{
* Correspondence: randall.holcombe@mssm.edu

${ }^{2}$ Tisch Cancer Institute of Mt. Sinai School of Medicine, New York, NY, USA

Full list of author information is available at the end of the article
} 


\section{Background}

Tumor infiltration by cells involved in the cellular immune response appears to correlate with prognosis for patients with colon cancer, suggesting that the immune system plays a critical role in patient outcome [1-3]. Following surgical resection of a colonic tumor, improved overall survival in patients with early stage (I-III) disease correlates with the extent of immune infiltration of the primary tumor with dendritic cells and T-cells. The reduced relapse rate in these patients suggests that sensitization to tumor antigens and an associated viable cellular immune response may eradicate microscopic residual disease. While patients with stage IV (metastatic) colorectal cancer are generally considered incurable, it is theoretically possible that stimulation of anti-tumor aspects of the immune system might positively affect response to therapy and overall median survival [4], analogous to the phenomenon noted for earlier stages of this disease. In addition, patients with advanced cancer are known to have tumor-related immune suppression and chemotherapy, typically administered in this setting, is also immunosuppressive $[5,6]$. However, the degree of immunosuppression of different chemotherapy regimens, and the precise effects of chemotherapy on specific lymphocyte subsets have not been rigorously defined. Still, for patients with colon cancer, reversing the innate immune suppression caused by any of the potential etiologies may be advantageous.

GM-CSF is frequently utilized in cancer patients to aid in hematopoietic recovery but it also has pleiotropic effects on components of the immune system, including being a requisite growth factor for myeloid dendritic cells (DCs) that play an important role in tumor antigen presentation and stimulation of immune responses [7]. Our hypothesis was that GM-CSF would be beneficial for patients with advanced colorectal cancer by stimulating cellular immune responses, potentially including Th1 immune responses, specifically by activating dendritic cells and also might abrogate chemotherapy induced immunosuppression common in this patient population. We devised a phase II trial augmenting standard chemotherapy with mid-cycle GM-CSF and monitored effects on the circulating dendritic cell and other peripheral blood mononuclear cell (PBMC) populations along with evaluation of Th1 type immune responses. The principal endpoints of this study were biologic in order to provide a potential rationale for larger studies with survival endpoints including GM-CSF.

\section{Materials \& methods}

\section{Clinical trial design and conduct}

Patients who were receiving chemotherapy for stage IV colorectal cancer were identified for this study. Enrollment for this study began in June, 2003 and ended January 2007, with 20 patients enrolled onto clinical trial
NCT00257322. The study was approved by the University of California, Irvine Institutional Review Board and all patients signed written informed consent prior to participation. All research was conducted in compliance with the Helsinki Declaration for ethical research in humans. Patients received $500 \mu \mathrm{g}$ GM-CSF daily for 4 days starting 24 hours after completion of each chemotherapy cycle. Initial patients received weekly 5 -fluorouracil with leucovorin $(n=12)$. With changes in standard of care that occurred during the conduct of the study, a protocol modification allowed enrollment of patients receiving biweekly mFOLFOX6 (5FU, leucovorin, oxaliplatin) chemotherapy $(\mathrm{n}=8)$. Blood was obtained before chemotherapy/GMCSF administration and 24 hours following the final dose of GM-CSF.

\section{Quantitative Real-time PCR (qRT-PCR)}

Blood samples drawn from the patients were immediately processed for the isolation of PBMCs using Histopaque 1083 (Sigma-Aldrich). Trizol (Invitrogen) was added to the PBMC pellets and stored at $4^{\circ} \mathrm{C}$ for subsequent RNA extraction. cDNA preparation for each RNA sample was performed using the high capacity cDNA reverse transcription kit (Applied Biosystems). PBMC expression of $\gamma$ interferon, T-bet transcription factor (Tbx21), DC-LAMP, and Wnt 5 a by quantitative real-time PCR was performed, the former two as measures of Th1 immunity. All experimental mRNA were normalized to the housekeeping gene $\beta$-actin mRNA.

\section{Flow cytometry}

Standard whole blood staining protocols were used to evaluate circulating leukocyte populations. Phlebotomy samples in EDTA anti-coagulated blood were collected and individual aliquots of $200 \mu \mathrm{l}$ of blood were placed into \#2059 tubes (BD Biosciences) and stained with the appropriate experimental or isotype control antibodies. Tubes were then incubated at room temperature (RT) for $60 \mathrm{~min}$ utes in the dark, with mixing every 15 minutes. RBCs were lysed with ACK lysis media for 15 minutes at RT and residual cells pelleted by centrifugation. Supernatant was discarded by aspiration, cells washed and the cell pellet was resuspended in either $500 \mu \mathrm{l}$ of staining media (if flow cytometry analysis is to be immediate) or $50 \%$ volume of staining media and $50 \%$ volume freshly prepared $4 \%$ paraformaldehyde. In the latter case tubes were stored at $4^{\circ} \mathrm{C}$ in the dark for no more than 48 hours before flow cytometry analysis. Calabrite 3, a calibration bead set (BD Biosciences) were used for standardization of the conditions and compensation controls for the three-color flow cytometry analysis according to manufacturer's instructions. Additional fluorophore controls, CD3-FITC, CD8-PE, CD4-TC, were used to verify the uniformity of conditions for the analyses. 


\section{Statistical Methods}

Expression of mRNA by real-time PCR was compared, pre- and post-treatment, with a Wilcoxin matched pairs signed rank test. Changes in lymphocyte subsets preand post-treatment were compared similarly. Differences across cycles of chemotherapy/GM-CSF administration were analyzed for statistical significance by a two-tailed unpaired t-test with Welch's correction.

\section{Results}

\section{Clinical trial}

Twenty patients were enrolled in the clinical trial and preand post-treatment (i.e., chemotherapy and GM-CSF) samples were evaluable for 16 patients. Samples were obtained ranging from 1 to 5 cycles with a median of 3 cycles ( 6 biologic sample time points) overall. There were no toxicities or adverse events to GM-CSF reported. Initially, most patients received weekly 5FU/leucovorin though later, because of changes that occurred in the standard of care, the protocol was amended to include patients receiving biweekly mFOLFOX6. No significant differences in immunologic endpoints were seen between the two groups. In addition, no patients were removed for study for hematologic toxicities and there was no increased toxicity beyond that seen in other trials with these specific chemotherapy agents without GMCSF. In all cases, patients received 4 daily dosages of GM-CSF starting 24 hours after completion of the chemotherapy cycle.

\section{Cellular immune response}

Cellular immune responses were quantified through the evaluation of expression of relevant genes from PBMCs by
qRT-PCR. Expression of mRNA for both DC-lamp, a transmembrane glycoprotein expressed on mature dendritic cells [8] and Wnt5a, which activates macrophages and T-cells through its interaction with the cell surface frizzled-5 receptor [9] were minimally detected in the patient samples (Figures not shown). $\gamma$-interferon mRNA levels remained unchanged with the administration of GM-CSF after chemotherapy (Figure 1A). $\gamma$-interferon is produced by Th1 cells, NK cells and, to a lesser extent, dendritic cells [10]. However, there was a statistically significant 1.7 fold decrease in T-bet transcription factor (Tbx21) mRNA following the administration of GM-CSF after chemotherapy ( $\mathrm{p}=0.043$; Figure $1 \mathrm{~B})$, implicating an overall decrease in Th1-type cellular immune response [11].

\section{Lymphocyte subsets and dendritic cells}

Individual lymphocyte subsets were evaluated by flow cytometry before and after the administration of chemotherapy and GM-CSF. The subsets that were evaluated include: CD4+, CD8 +, CD4+CD25 high (Treg) and CD56 $+\mathrm{CD} 3+(\mathrm{NK}-\mathrm{T})$ populations. Values represent average percentages of nucleated cells in whole blood samples $(\mathrm{n}=$ 23). Significant differences were evaluated with a twotailed Wilcoxon Signed rank test. The decrease in CD4 and CD8 T lymphocyte subsets was statistically significant at $\mathrm{p}=0.0064$ and $\mathrm{p}=0.0012$ respectively. The decrease in the Treg population was also significant at $\mathrm{p}=0.0042$ and the decrease in NK-T cells approached statistical significance of $\mathrm{p}=0.0997$ (Figure 2A).

In the first and subsequent cycles of chemotherapy and GM-CSF the percent change in lymphocyte subsets that include CD4+, CD8 +, CD4+CD25 high (Treg) and
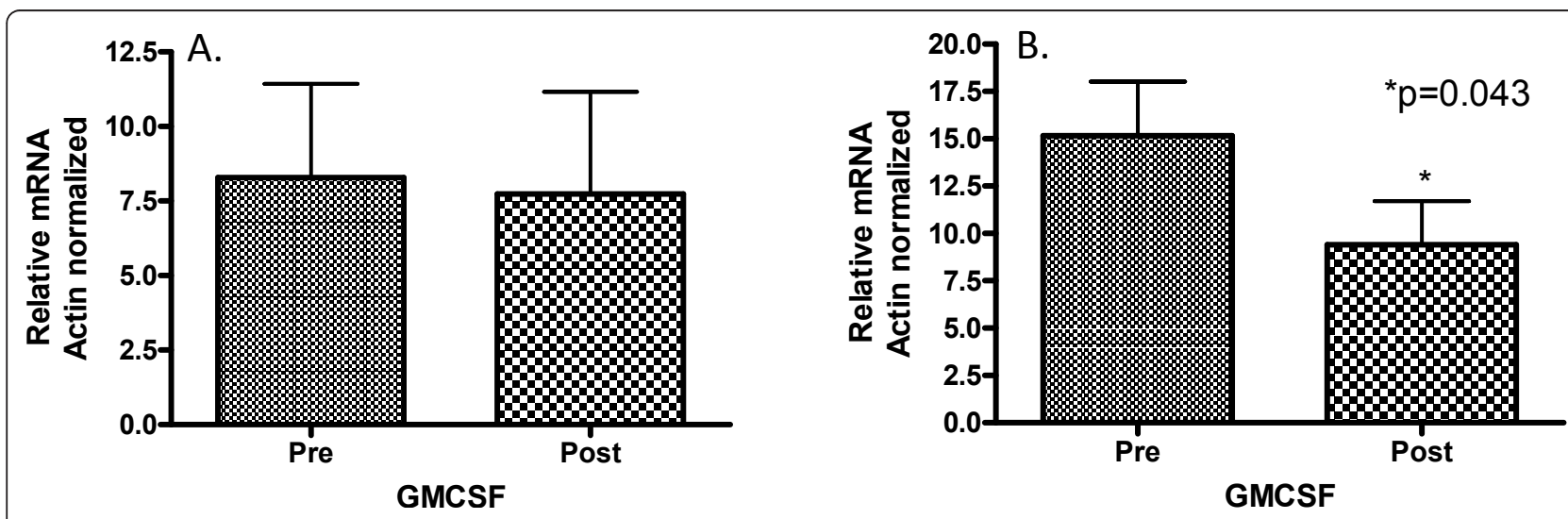

Figure 1 Panel A. Levels of $\boldsymbol{\gamma}$-interferon mRNA following the administration of GM-CSF after chemotherapy. $\gamma$-interferon mRNA levels were measured in 16 patients pre-chemotherapy/GM-CSF administration and post-GM-CSF/Chemotherapy administration by quantitative real time PCR of peripheral blood mononuclear cells. $\gamma$-interferon mRNA levels were normalized against the housekeeping gene $\beta$-actin mRNA. There was no statistically significant change between the two groups. Panel B. Levels of T-bet transcription factor mRNA following the administration of GM-CSF after chemotherapy. T-bet transcription factor mRNA levels were measured in 16 patients pre-chemotherapy/GM-CSF administration and post-GMCSF/chemotherapy administration by quantitative real time PCR of peripheral blood mononuclear cells. T-bet transcription factor mRNA levels were normalized against the housekeeping gene $\beta$-actin mRNA. There was an approximate 1.7 fold decrease in T-bet transcription factor mRNA levels following the administration of GM-CSF after chemotherapy ( $p=0.043$ ). The statistical significance was determined by a Wilcoxin matched pairs test. 

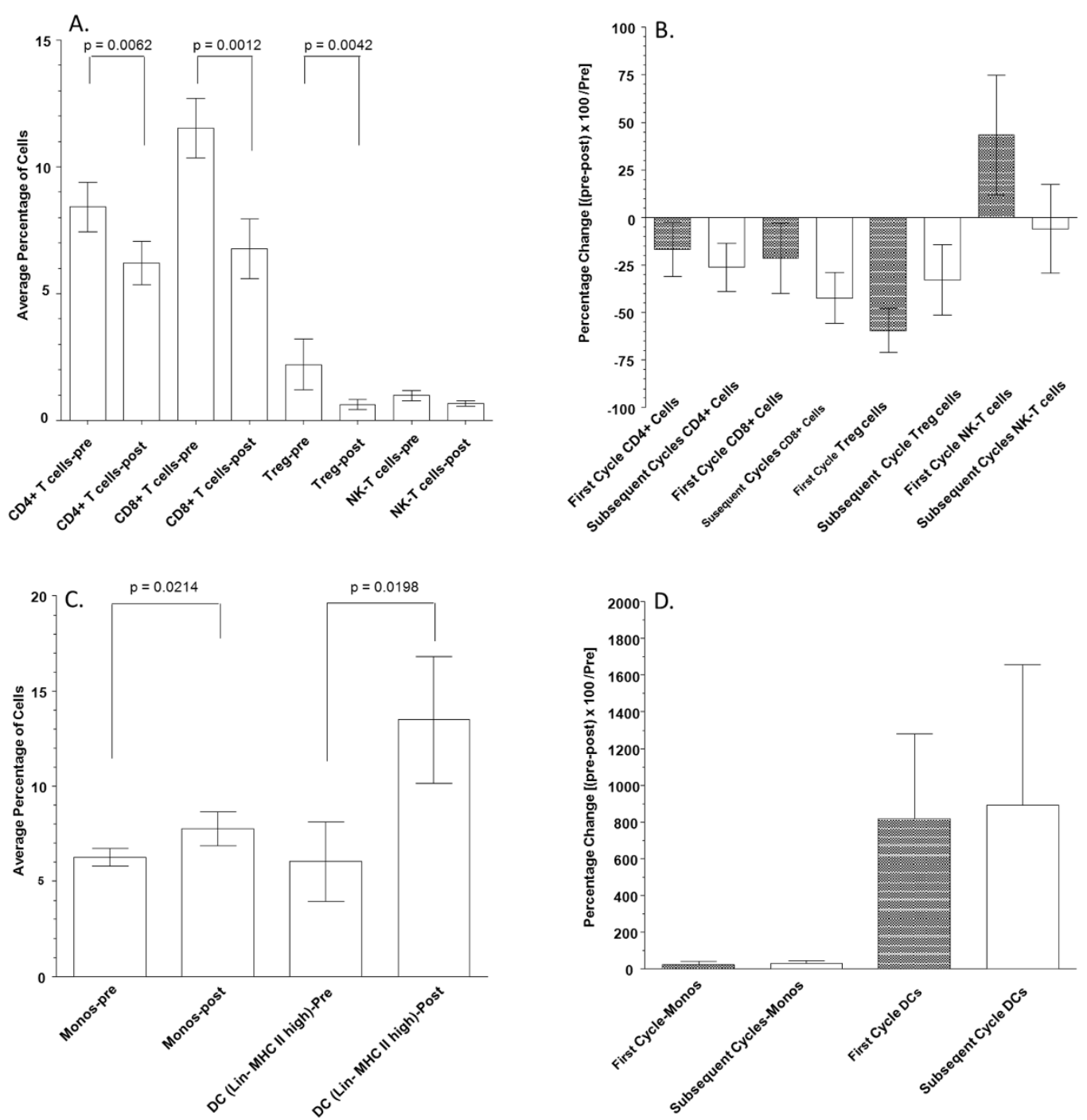

Figure 2 Panel A. Changes in lymphocyte subsets associated with chemotherapy and GM-CSF administration. Individual lymphocyte subsets were evaluated by flow cytometry before and after the administration of chemotherapy and GM-CSF. The subsets that were evaluated include: CD4+, CD8 +, CD4+CD25 high (Treg), CD56+ CD3+ (NK-T) populations. Values depicted represent average percentages of nucleated cells in whole blood samples $(n=23)$ with error bars depicting standard error values. Significant differences were evaluated with a Wilcoxon matched pairs test. The decrease in CD4 and CD8 T lymphocyte subsets was statistically significant at $p=0.0064$ and $p=0.0012$ respectively. The decrease in the Treg population was also significant at $p=0.0042$ while the decrease in NK-T cells approached statistical significance $p=$ 0.0997. Panel B. Percent change in lymphocyte subsets across multiple cycles of chemotherapy \& GM-CSF. In the first and subsequent cycles of Chemotherapy \& GM-CSF the percent change in lymphocyte subsets that include: CD4+, CD8 +, CD4+CD25 high (Treg), CD56+ CD3+ (NK-T) populations, were determined. Percent change was calculated in the standard fashion [(pre- treatment value - post-treatment value)/pretreatment value] $\times 100$. None of the differences in lymphocyte subset changes between the first and subsequent cycles reached statistical significance by two-tailed unpaired t-test with Welch's correction. Panel C. Changes in monocytes and myeloid DCs associated with chemotherapy and GM-CSF administration. Individual cell populations were evaluated by flow cytometry before and after the administration of chemotherapy and GM-CSF. Monocyte populations were identified as being CD14+. Myeloid dendritic cells (DCs) were identified using a lineage cocktail (CD3, CD20, CD14, CD56) negative cells that had MHC II high expression. Values depicted represent average percentages of nucleated cells in whole blood samples $(n=23)$ with error bars depicting standard error values. Differences were evaluated with a Wilcoxon matched pairs test. Differences in monocytes and DCs were statistically significant $p=0.0214$ and $p=0.0198$ respectively. Panel $D$. Percent change in monocytes and dendritic cells across multiple cycles of chemotherapy and GM-CSF. In the first and subsequent cycles of Chemotherapy \& GMCSF the percent change in monocytes (CD14+ cells) and dendritic cells (DCs) [lineage cocktail (CD3, CD20, CD14, CD56) negative, MHC II high expression] were determined. Percent change was calculated in the standard fashion [(pre-treatment value - post-treatment value)/pre-treatment value] $\times 100$. None of the differences in lymphocyte subset changes between the first and subsequent cycles reached statistical significance by two-tailed unpaired t-test with Welch's correction. 
CD56+ CD3+ (NK-T) populations were determined. Percent change was calculated in the standard fashion [(Pre treatment value-Post treatment value)/pre-treatment value] $\times 100$. None of the differences in lymphocyte subset changes between the first and subsequent cycles reached statistical significance by two-tailed unpaired $t$ test with Welch's correction (Figure 2B).

Individual cell populations were evaluated by flow cytometry before and after the administration of chemotherapy and GM-CSF. Monocyte populations were identified as being CD14+. Myeloid DCs were identified using a lineage cocktail (CD3, CD20, CD14, CD56) negative cells that had MHC II high expression. Values represent average percentages of nucleated cells in whole blood samples $(n=23)$. There were statistically significant increases in both monocytes $(p=0.0214)$ and DCs $(p=0.01980)$ following chemotherapy and GM-CSF (Figure 2C). Overall, there was no difference between the first and subsequent cycles of chemotherapy and GM-CSF in the percent change in monocytes (CD14+ cells) and DCs (lineage cocktail (CD3, CD20, CD14, CD56) negative, MHC II high expression; Figure 2D). However, approximately $50 \%$ of the patients exhibited increased dendritic cell numbers in subsequent chemotherapy/GM-CSF cycles.

\section{Discussion}

GM-CSF is utilized primarily for its activity in increasing granulocyte production following administration of bone marrow suppressive cytotoxic chemotherapy. However, largely because of its role in stimulating monocytic precursors, it has long been recognized that it possesses immunostimulatory activity [7]. Our utilization of GM-CSF in this trial was to attempt to abrogate the immunosuppression induced by chemotherapy [5] and by the cancer itself [6]. In this population of patients receiving chemotherapy for metastatic colorectal cancer, GM-CSF was administered without significant toxicity.

GM-CSF did not augment cellular, Th1, immune functioning, as measured by the methodologies utilized in this study. The reduction in T-bet is postulated to be secondary to chemotherapy. The lack of concomitant reduction of $\gamma$-interferon may be due to GM-CSF-dependent stimulation of dendritic cells, which can also produce this cytokine. Together with the decreases in the CD4+, CD8+, CD14+ and CD56 =/CD3+ populations, the decrease in Tbet suggests that there was not selective sparing of Th1 biased lymphocytes by the cytotoxic chemotherapy to account for the stable $\gamma$-interferon mRNA expression in PBMCs. As there was no control arm of patients receiving chemotherapy without GM-CSF on this study, we cannot quantitate the absolute reduction in PBMC $\gamma$-interferon production which might be seen following chemotherapy alone. With respect to $\mathrm{T}$-cell subset changes, approximately $2 / 3(66 \%)$ of subjects had a significant decrease in
CD4+ lymphocytes and approximately $80 \%$ of subjects had a significant decrease in CD8+ lymphocytes with each cycle.

One of the most significant findings from this clinical trial was the increase in the number of circulating myeloid DCs (Lineage-, MHC high). These increased in approximately $75 \%$ of subjects across all cycles. In subsequent cycles of chemotherapy and GM-CSF, approximately 50\% of the subjects experienced increased DC numbers over the initial cycle. Dendritic cells are potent antigen presenting cells and play a pivotal role in the induction of immune responses. Dendritic cells have been directly implicated in anti-tumor immune responses [12-15]. The increase in circulating DCs, likely a consequence of GMCSF administration, may contribute to the anti-tumor effects of chemotherapy administered in the adjuvant or metastatic setting.

Another important observation was the reduction in regulatory T-cells (Treg) following chemotherapy/GMCSF. Approximately $90 \%$ of subjects had a significant (> $20 \%)$ decrease in Treg lymphocytes with the first cycle. Tregs can suppress the activity of cytotoxic $\mathrm{T}$ cells through direct cell-to-cell contact or via the release of cytokines such as transforming growth factor beta. Depletion of intratumoral Tregs has been shown to enhance antitumor immunity and promote tumor rejection in mouse models [16]. In patients with colon cancer, increased numbers of Treg cells has been associated with a poor clinical prognosis [17]. This decrease in circulating Treg cells argues against a significant increase in myeloid derived suppressor cells, implicated in the induction of Tregs [18], as a result of the administration of GM-CSF; an effect that has been inconsistently reported in other settings[19-22]. Thus, the reduction in Tregs seen in our study, and the concomitant loss of immune system suppression that these cells induce, may be prognostically favorable.

As noted previously, this study did not contain a chemotherapy only control arm. Therefore, the effects of chemotherapy cannot be distinguished specifically from the effects of GM-CSF. Still, in patients with metastatic colorectal cancer receiving chemotherapy and GM-CSF, cellular immune responses are inhibited and the impairment in Th1 type immune parameters are not abrogated by administration of GM-CSF. However, administration of GM-CSF along with chemotherapy does lead to an increase in circulating DCs and a reduction in inhibitory Tregs. Together, this may provide a mechanism for augmentation of innate immune responses and may provide benefit to colorectal cancer patients receiving chemotherapy.

\section{Conclusions}

This phase II clinical trial confirms that chemotherapy in patients with metastatic colorectal cancer suppresses 
Th1 cellular immunity but demonstrates that mid-cycle administration of GM-CSF can significantly increase the proportion of circulating dendritic cells and reduce the proportion of circulating Treg cells. Th1 activity, as measured by $\gamma$-interferon production, was not affected. These finding suggest that GM-CSF can abrogate important aspects of the immune suppression seen in patients with advanced colorectal cancer and may provide a non-toxic intervention to augment anti-tumor immunity for cancer patients receiving cytotoxic chemotherapy.

\section{Acknowledgements}

Supported in part from an investigator-initiated grant from Berlex Corporation and from seed funding provided by the Chao Family Comprehensive Cancer Center. The authors wish to thank Serena Bhela for assistance with immunologic assays. Dr. Nelson and Dr. Holcombe served as co-senior investigators for this research.

\section{Author details}

'Division of Hematology/Oncology, University of California, Irvine, CA, USA. ${ }^{2}$ Tisch Cancer Institute of Mt. Sinai School of Medicine, New York, NY, USA.

\section{Authors' contributions}

MM, NO, MP and KP conducted immunologic assays including PCR and flow cytometry. MM assisting in maintaining clinical trial related data according to IRB guidelines. ELN and RFH conceived this study, provided oversight for all aspects of the laboratory investigation, were involved in obtaining informed consent and providing treatment for patients and directed the overall project. All authors were involved in manuscript preparation.

\section{Competing interests}

The authors declare that they have no competing interests.

Received: 23 September 2011 Accepted: 23 January 2012

Published: 23 January 2012

\section{References}

1. Menon AG, et al: Immune system and prognosis in colorectal cancer: a detailed immunohistochemical analysis. Lab Invest 2004, 84(4):493-501.

2. Atreya I, Neurath MF: Immune cells in colorectal cancer: prognostic relevance and therapeutic strategies. Expert Rev Anticancer Ther 2008, 8(4):561-72.

3. Mlecnik B, et al: Histopathologic-based prognostic factors of colorectal cancers are associated with the state of the local immune reaction. $J$ Clin Oncol 2011, 29(6):610-8.

4. Rao B, et al: Clinical outcomes of active specific immunotherapy in advanced colorectal cancer and suspected minimal residual colorectal cancer: a meta-analysis and system review. J Trans/ Med 2011, 9(1):17.

5. Rasmussen $L$, Arvin A: Chemotherapy-induced immunosuppression. Environ Health Perspect 1982, 43:21-5.

6. Whiteside TL: Immune suppression in cancer: effects on immune cells, mechanisms and future therapeutic intervention. Semin Cancer Biol 2006, 16(1):3-15

7. Metcalf $D$ : The molecular biology and functions of the granulocytemacrophage colony-stimulating factors. Blood 1986, 67(2):257-67.

8. Arruda $L B$, et al: Dendritic cell-lysosomal-associated membrane protein (LAMP) and LAMP-1-HIV-1 gag chimeras have distinct cellular trafficking pathways and prime $T$ and $B$ cell responses to a diverse repertoire of epitopes. J Immunol 2006, 177(4):2265-75.

9. Blumenthal A, et al: The Wingless homolog WNT5A and its receptor Frizzled-5 regulate inflammatory responses of human mononuclear cells induced by microbial stimulation. Blood 2006, 108(3):965-73.

10. Frucht DM, et al: IFN-gamma production by antigen-presenting cells: mechanisms emerge. Trends Immunol 2001, 22(10):556-60.
11. Wang J, et al: Transcription factor T-bet regulates inflammatory arthritis through its function in dendritic cells. J Clin Invest 2006, 116(2):414-21.

12. Lapteva $\mathrm{N}$, et al: Attraction and activation of dendritic cells at the site of tumor elicits potent antitumor immunity. Mol Ther 2009, 17(9):1626-36.

13. Taieb J, et al: A novel dendritic cell subset involved in tumor immunosurveillance. Nat Med 2006, 12(2):214-9.

14. Petersen TR, et al: Potent anti-tumor responses to immunization with dendritic cells loaded with tumor tissue and an NKT cell ligand. Immunol Cell Biol 2010, 88(5):596-604.

15. Song YC, et al: Presentation of lipopeptide by dendritic cells induces anti-tumor responses via an endocytosis-independent pathway in vivo. $J$ Leukoc Biol 2011.

16. Koch $\mathrm{M}$, et al: Tumor infiltrating $\mathrm{T}$ lymphocytes in colorectal cancer: Tumor-selective activation and cytotoxic activity in situ. Ann Surg 2006, 244(6):986-92, discussion 992-3.

17. Salama $\mathrm{P}$, et al: Tumor-infiltrating FOXP3+ T regulatory cells show strong prognostic significance in colorectal cancer. J Clin Oncol 2009, 27(2):186-92.

18. Condamine T, Gabrilovich DI: Molecular mechanisms regulating myeloidderived suppressor cell differentiation and function. Trends Immunol 2011, 32(1):19-25.

19. Anthony DD, et al: Lower peripheral blood CD14+ monocyte frequency and higher CD34+ progenitor cell frequency are associated with HBV vaccine induced response in HIV infected individuals. Vaccine 2011, 29(19):3558-63.

20. Daud Al, et al: Phenotypic and functional analysis of dendritic cells and clinical outcome in patients with high-risk melanoma treated with adjuvant granulocyte macrophage colony-stimulating factor. J Clin Oncol 2008, 26(19):3235-41.

21. Filipazzi $P$, et al: Identification of a new subset of myeloid suppressor cells in peripheral blood of melanoma patients with modulation by a granulocyte-macrophage colony-stimulation factor-based antitumor vaccine. J Clin Oncol 2007, 25(18):2546-53.

22. Kaufman $\mathrm{HL}$, et al: Local and distant immunity induced by intralesional vaccination with an oncolytic herpes virus encoding GM-CSF in patients with stage IIIc and IV melanoma. Ann Surg Oncol 2010, 17(3):718-30.

doi:10.1186/1475-2867-12-2

Cite this article as: Martinez et al.: Granulocyte-macrophage stimulating factor (GM-CSF) increases circulating dendritic cells but does not abrogate suppression of adaptive cellular immunity in patients with metastatic colorectal cancer receiving chemotherapy. Cancer Cell International 2012 12:2.

\section{Submit your next manuscript to BioMed Central and take full advantage of:}

- Convenient online submission

- Thorough peer review

- No space constraints or color figure charges

- Immediate publication on acceptance

- Inclusion in PubMed, CAS, Scopus and Google Scholar

- Research which is freely available for redistribution 